\title{
ПАТОЛОГИЯ ЩИТОВИДНОЙ ЖЕЛЕЗЫ У ДЕТЕЙ С СИНДРОМОМ ШЕРЕШЕВСКОГО-ТЕРНЕРА
}

'Песковая Н.А., ${ }^{2}$ Солнцева А.В.

\begin{abstract}
'ГУ «Республиканский чентр медицинской реабилитации и бальнеолечения», Минск, Республика Беларусь ${ }^{2}$ Белорусский государственный медицинский университет, Минск, Республика Беларусь
\end{abstract}

ЦЕЛЬ: оценить распространенность патологии щитовидной железы у детей с синдромом Шерешевского-Тернера с разными вариантами кариотипа.

МАТЕРИАЛЫ И МЕТОДЫ: проведен ретроспективный анализ медицинской документации 92 пациенток с синдромом Шерешевского-Тернера (СШТ) в возрасте от 1 до 18 лет, наблюдавшихся в ГУ «Республиканский центр медицинской реабилитации и бальнеолечения» в 2005-2019 гг. В зависимости от варианта кариотипа пациентки разделены на 4 группы: 1-я - девочки с моносомией X (n=47, 51,1\%), 2-я - с мозаичным вариантом кариотипа 45,X/46XX (n=14, 15,2\%), 3-я - с присутствием изохромосомы $X$ по длинному плечу - iso-Xq $(n=14,15,2 \%)$, 4-я - со структурными аномалиями $X$ хромосомы ( $n=17$, 18,5\%). Оценка тиреоидной функции проводилась всем девочкам при первичном обращении к эндокринологу и контролировалась В динамике. Проанализированы показатели уровней тиреотропного гормона (ТТГ), свободного тироксина (Т4 св), антител к тиреоидной пероксидазе (АТ к ТПО) в сыворотке крови, результаты ультразвукового исследования (УЗИ) щитовидной железы. Статистическая обработка полученных данных проведена с использованием программного пакета SPSS Statistics 22.0 и Microsoft Office Excel 2007.

PЕЗУЛЬТАТЫ: исследование тиреоидной функции показало, что у большего количества девочек с СШТ ( $\mathrm{n}=46,50 \%)$ выявлен субклинический гипотиреоз (ТТГ 6,1 [4,78;7,37] мкMЕ/мл), у 40,2\% девочек (n=37) наблюдалась нормальная функция ЩЖ с уровнем ТТГ 2,5 [2,05;3,58] мкМЕ/мл. Манифестный гипотиреоз выявлен у 6 пациенток (6,5\%) (ТТГ 20,0 [12,2;33] мкМЕ/мл), гипотироксинемия у 2 девочек (2,2\%), вторичный гипотиреоз в составе множественной врожденной гипофизарной недостаточности у одного ребенка (1,1\%). Случаев болезни Грейвса в анализируемой группе пациенток не зафиксировано. Диагностически значимый уровень АТ к ТПО отмечен у 27,2\% (n=25) девочек с СШТ, что подтверждает высокую частоту аутоиммунных заболеваний при этом синдроме. Среди девочек с повышенным уровнем АТ к ТПО гипофункция щитовидной железы выявлена у $80 \%(n=20)$, из них манифестный гипотиреоз - у 3 детей (12\%). Положительные антитиреоидные антитела зафиксированы у 37,6\% ( $\mathrm{n=17)}$ девочек с субклиническим гипотиреозом. Максимальное количество пациенток с повышенным уровнем АТ к ТПО отмечено в возрасте 11-14 лет ( $n=11,44 \%)$, что совпадает с возрастом наиболее частого выявления гипофункции щитовидной железы. Диагностически значимый уровень АТ к ТПО чаще зарегистрирован у девочек со структурными аномалиями X хромосомы $(\mathrm{n}=6,35,3 \%)$, реже - с кариотипом 45,X/46,XX (n=3, 21,4\%). У пациенток с моносомией X положительные антитиреоидные антитела выявлены в 25,5\% случаев (n=12), с iso-Xq - в 28,6\% $(\mathrm{n}=4)$.

ВЫВОДЫ: по результатам исследования установлено, что нарушение тиреоидной функции является частой эндокринной патологией при СШТ и встречается у 59,6\% пациенток в детском возрасте. Повышенный уровень АТ к ТПО выявлен у 27,2\% обследованных пациенток и в большинстве случаев привел к развитию гипотиреоза. Достоверной связи между возникновением патологии щитовидной железы и вариантом кариотипа при СШТ не установлено. Результаты исследования обосновывают необходимость проведения у пациенток детского возраста с СШТ скрининга для ранней диагностики тиреоидной патологии и своевременного назначения адекватной терапии.

КЛЮЧЕВЫЕ СЛОВА: синдром Шерешевского-Тернера, дети, щитовидная железа, гипотиреоз. 